\title{
A Case of Blue Nevus Mimicking Malignant Melanoma Within a Keloid Scar
}

Ecem Bostan', [MD]

ORCID: 0000-0002-8296-4836

Basak Yalici-Armagan', [MD]

ORCID: 0000-0001-9745-1331

Ozay Gokoz ${ }^{2}$, [MD]

ORCID: 0000-0001-9537-1127

Aysen Karaduman', [MD]

ORCID: 0000-0002-4056-6303

\section{'Department of Dermatology and Venereology, Hacettepe University, Faculty of Medicine, Ankara, Turkey. \\ ${ }^{2}$ Department of Pathology, Hacettepe University, Faculty of Medicine, Ankara, Turkey.}

Corresponding Author: Ecem Bostan Hacettepe University, Faculty of Medicine, Department of Dermatology and Venereology, Ankara, Turkey. Phone: +90 3123054127

E-mail: bostanecem@gmail.com

\section{n ABSTRACT Con}

Blue nevus is a type of melanocytic nevus which clinically presents itself as blue/gray plaque or nodule. It most commonly develops upon distal extremities especially dorsal hands and feet, trunk and scalp. Histopathologically, heavy melanocyte aggregations in the deep dermis result in blue nevus formation. Different types of blue nevus include common blue nevus, cellular blue nevus, plaque-type blue nevus, combined blue nevus, desmoplastic blue nevus and subungal blue nevus. Although clinical findings may be sufficient to diagnose blue nevus, dermoscopy could be used as a helpful tool to aid in reaching the correct diagnosis. Dermoscopy usually shows diffuse bluegray structureless area but since atypical clinical and dermoscopical features may be observed, histopathological diagnosis is the definitive step to differentiate blue nevus from its mimickers such as malignant melanoma. We want to present an interesting case of blue nevus mimicking malignant melanoma developing in a scar.

Keywords: Blue nevus, malignant melanoma, scar.

https://doi.org/10.32552/2021.ActaMedica.541

Received: 20 November 2020, Accepted: 18 March 2021,

Published online: 5 June 2021

\section{INTRODUCTION}

Blue nevus is an acquired or congenital form of melanocytic nevus with prominent blue/ steelgray pigmentation which results from the aggregation of melanocytes in the deep dermis [1]. Morphologically and dermoscopically, blue nevus may simulate malignant melanoma (MM) which can cause potential diagnostic pitfall [2]. Histopathological diagnosis is the substantial and definitive step to differentiate common blue nevus from blue nevi variants, MM or cutaneous metastasis of MM. Herein, a case of blue nevus simulating $\mathrm{MM}$ in association with a keloid scar is presented.

\section{CASE PRESENTATION}

A 34-year old woman was appointed to our outpatient clinic with the complaint of gradually growing gray-black plaque in association with a keloid scar on the left upper arm. Medical history revealed that scar was formed ten years ago with an unknown etiology. Patient didn't report any history of surgical operation. There was no personal or family history of melanoma. Approximately 2 months ago, a pinpoint-sized black papule started to appear just near the keloid scar. It turned into a steel-gray/blue, well-defined, hemorrhagic-crusted plaque in association with the keloid scar (Figure 1A). Dermoscopic examination revealed blue- 

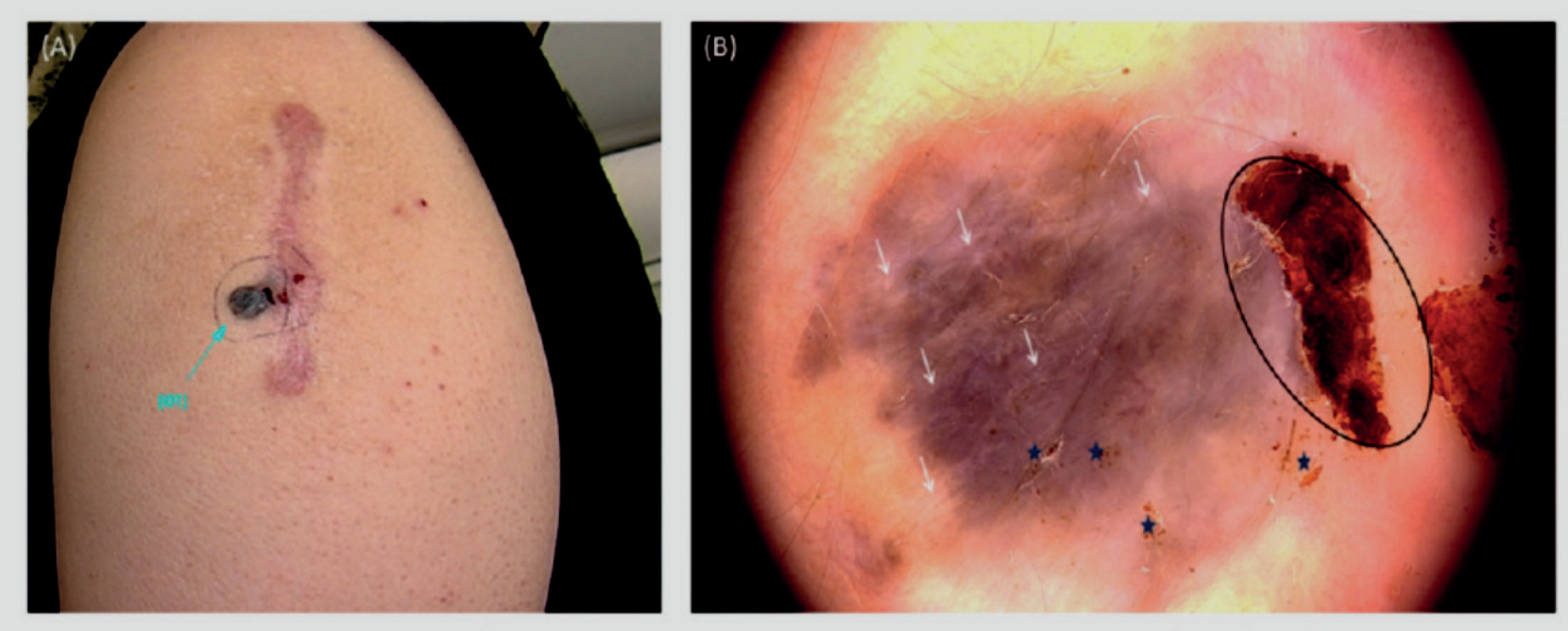

Figure 1. (A) A steel-gray/black, well-defined, hemorrhagic-crusted plaque in association with the keloid scar (blue arrow). (B) Dermoscopy revealed blue-white veil (white arrows), red/brown dots and clods (blue asterisks) on a diffuse steel-gray/dark blue structureless area with a big hemorrhagic crust (big circle) encircling the left border of the lesion.

white veil, red/brown dots and clods on a diffuse steel-gray/dark blue structureless area with a hemorrhagic crust encircling the left border of the lesion (Figure 1B). Our initial diagnosis was MM due to atypical clinical and dermoscopical features of the mentioned lesion. However, histopathological examination revealed a plaque type of deep blue nevus. The ulcer described clinically was situated on the scar tissue adjacent to the nevus (Figure 2A). The dendritic nevoid cells with melanophages (Figure 2B) and coarse collagen fibers were present predominantly in the dermis (Figure $3 \mathrm{~A}$ ). At the center, a small part of protrusion to the beginning of subcutaneous tissue was seen (Figure 2B, 3B). At this deep part, the adnexal structures along with erector pili muscle and peripheric nerve branches were entangled with the nevoid cells. The cellularity was not that much of a cellular blue nevus. Although the pattern looked like a deep penetrating nevus, the deep part did not reveal any pleomorphism

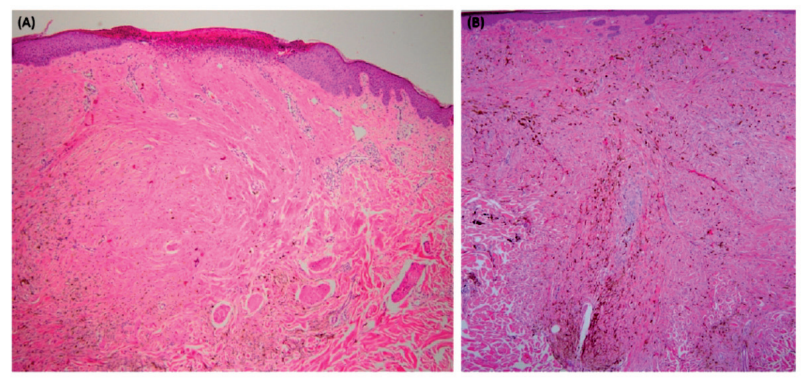

Figure 2. (A) Small ulcer on the scar tissue adjacent to the nevus. H.E. x40. (B) The dendritic nevoid cells throughout the dermis a small protrusion to the beginning of the subcutaneous tissue. H.E. x40. or mitoses. MelanA and HMB45 staining revealed diffuse positivity whereas S100 was positive only focally (Figure 4). Ki-67 proliferation index was very low. A large and deep blue nevus, plaque type was the final diagnosis.

\section{DISCUSSION}

There is only one case report in the literature related to malignant blue nevus in association with a keloid scar in an African American patient [3]. This case underlined the importance of routine histopathological examination of excised keloid scars in order to miss any concurrent malignant conditions [3]. To our knowledge, our case is the first example of blue nevus occurring in association
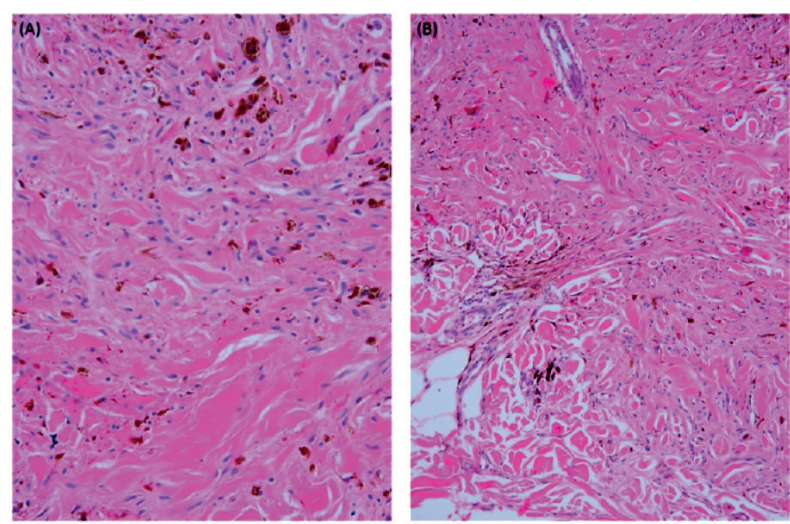

Figure 3. (A) Nevoid cells interspersed among coarse collagen fibers. H.E. x 200. (B) Hypocellular deep area without pleomorphism or mitoses. H.E. x100. 


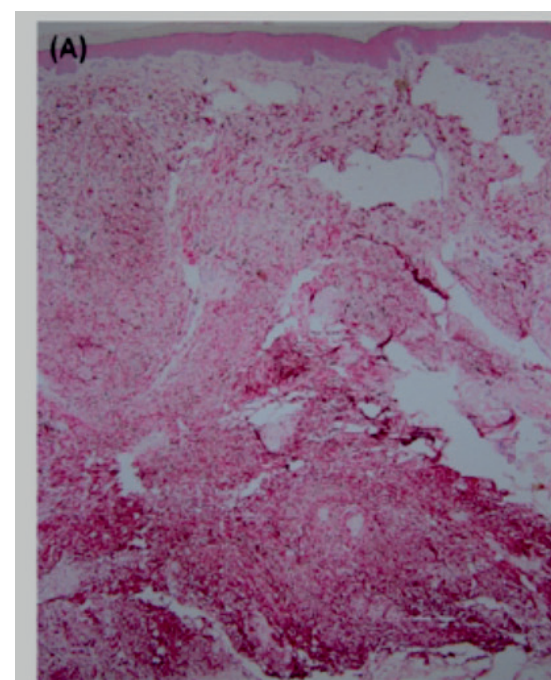

Melan A

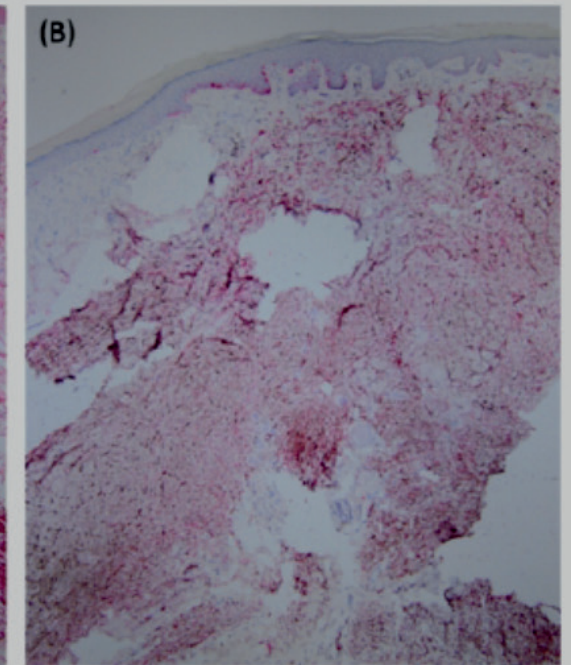

HMB 45

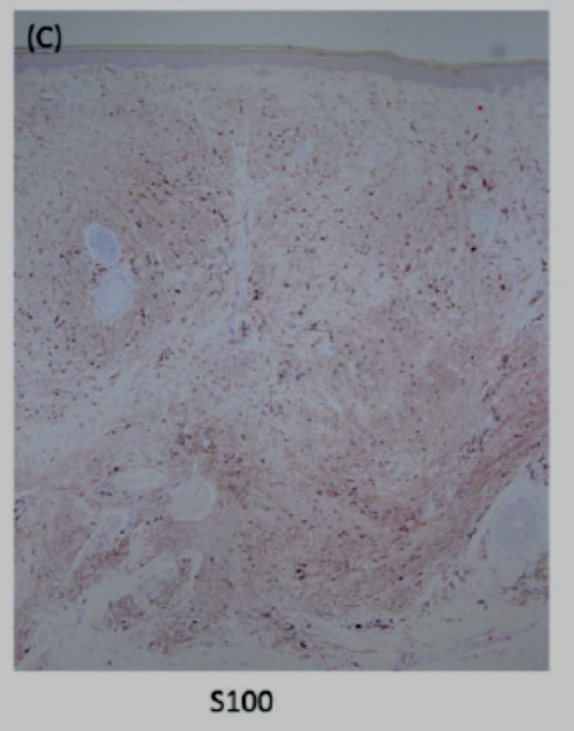

Figure 4. (A, B) MelanA and HMB45 staining revealed diffuse positivity whereas S100 was positive only focally(C).

with a keloid scar in a Caucasian patient. Blue nevus may be a great simulator of MM clinically, especially when presents with satellite lesions [4]. Likewise, current case simulated $\mathrm{MM}$ due to its atypical clinical presentation and rapid growth pattern. The patient also reported symptoms such as pain, tingling sensation, numbness and edema of the left arm arising suspicion for a cutaneous malignancy. Dermoscopically, blue nevi are described to present with homogeneous blue, black or gray pigmentation pattern along with focal white scarlike depigmentation, streaks, dots or globules [1]. Our case's dermoscopic examination revealed a diffuse gray-blue structureless area along with blue-white veil which is generally considered as MM associated dermoscopic criteria [5].
We present an interesting case of blue nevus developing within a keloid scar. Although distinguishing blue nevi from blue nevi variants and $\mathrm{MM}$ can be challenging already, the rapid

growing pattern of the lesion and dermoscopic finding of blue white veil make the diagnose more difficult in the current case. Consequently, histopathological examination is essential in

such conflicting cases to determine the correct diagnosis.

\section{CONFLICT Of INTEREST}

The authors declare that there is no conflict of interests.

\section{weO REFERENCES Cen}

[1] Di Cesare A, Sera F, Gulia Aet al. The spectrum of dermatoscopic patterns in blue nevi. J Am Acad Dermatol 2012; 67: 199-205.

[2] Sardoy A, Bidabehere MB, Gubiani ML et al. Blue Nevus with Satellite Lesions Mimicking Malignant Melanoma. Actas Dermosifiliogr 2018; 109; 377-378.

[3] Theopold C, Pritchard S, McGrouther DA et al. Keloid scar harbouring malignant blue naevus emphasises the need for excision biopsy and routine histology. J Plast Reconstr Aesthet Surg 2009; 62:93-95.
[4] Yonei N, Kimura A, Furukawa F et al. Common blue nevus with satellite lesions needs a differential diagnosis from malignant melanoma. Case Rep Dermatol 2013; 5; 244247.

[5] Ciudad-Blanco C, Avilés-Izquierdo JA, Lázaro-Ochaita $P$ et al. Dermoscopic findings for the early detection of melanoma: an analysis of 200 cases. Actas Dermosifiliogr 2014; 105; 683-693. 